\title{
Directional model analysis of the spectral reflection from the fovea and para-fovea
}

Tos T. J. M. Berendschot

University Eye Clinic Maastricht

PO Box 5800

NL-6202 AZ Maastricht, The Netherlands

\author{
Jan van de Kraats \\ Martijn J. Kanis \\ Dirk van Norren \\ University Medical Center Utrecht \\ Department of Ophthalmology \\ NL-3508 GA Utrecht, The Netherlands
}

\begin{abstract}
Directional and nondirectional spectral reflection data from $0,1,2,4$, and 8 deg eccentricity, and the optic disk, were analyzed from 400 to $950 \mathrm{~nm}$ with an existing optical reflection model. The optical model, developed for the fovea, appeared to be also suitable for more eccentric locations. The optical densities of melanin and of the macular pigments zeaxanthin and lutein peaked in the fovea, in correspondence with literature data. The amplitude of the directional component, originating in the cone photoreceptors, had its maximum at $1 \mathrm{deg}$. The maximum of the directionality (peakedness) occurred at a slightly higher eccentricity. $\odot 2010$ Society of Photo-Optical Instrumentation Engineers. [DOI: 10.1117/1.3523370]
\end{abstract}

Keywords: fundus reflectance; macular pigment; lutein; zeaxanthin; Stiles-Crawford; photoreceptor.

Paper 10384R received Jul. 8, 2010; revised manuscript received Sep. 16, 2010; accepted for publication Oct. 15, 2010; published online Dec. 15, 2010.

\section{Introduction}

When fundus reflectometry was introduced in 1952, it was aimed at studying the macular pigment ${ }^{1}$ and elucidating the spectral absorption characteristics and the kinetics of the visual pigments in the photoreceptors..$^{2-4}$ It was immediately realized that the interpretation of the data was hampered by the lack of knowledge of the pathways of light in the retina. This was at first called the "stray light" issue, and rather crude models of the receptor layer, without properties of anterior and posterior layers, were subsequently developed. ${ }^{3,5,6}$ In 1986, a model was proposed aimed at explaining the spectral reflection from the fundus at the fovea, the para-fovea and the optic disk. It included the percentage reflection at the inner limiting membrane (ILM), the retinal pigment epithelium (RPE), and at the sclera, and yielded the optical densities of the lens, the macular pigment, blood, and melanin. ${ }^{7}$ This model, however, ignored the photoreceptor layer, with the (only partly correct) argument that the visual pigments were fully bleached. Measurements at that time had a limited number of spectral points and lacked directional information. In 1989, a model was presented, based on detailed spectra, providing a more complex description of the layers posterior to the, again ignored, photoreceptor layer. ${ }^{8}$ Models based on inverse Monte Carlo simulation were also used for estimating optical properties of absorbers in the retina..$^{9,10}$

In 1996, the first model appeared that included the cone photoreceptor layer, taking account of both spectral properties of photo pigments and directional reflection. The directional reflection, termed the optical Stiles-Crawford effect (SCE) causes a Gaussian-shaped light distribution in the pupil plane. ${ }^{11-16}$ A great advantage of incorporating the directionality is that it helps in distinguishing between the different retinal layers. In the directional reflection, only absorption anterior to the photoreceptor layer is present (i.e., in the eye media and in

Address all correspondence to: Tos T. J. M. Berendschot, University Eye Clinic Maastricht, P.O. Box 5800, NL-6202 AZ Maastricht, The Netherlands; Tel: + 3143-3877345; fax: +31-43-3975343; E-mail: t.berendschot@ohk.unimaas.nl macular pigment). Absorption in blood and melanin, located in layers posterior to the receptor layer, leaves no spectral imprint on it. Recently, this foveal model was updated. ${ }^{17}$ The neutral reflection from the cones was replaced by a $\lambda^{-2}$ wave guiding behavior, ${ }^{18,19}$ and a wedged shaped layer of blood was used to better model the different lengths of the pathways through small and large blood vessels. The model also included recapture of light in the deeper retinal layers lost earlier by Rayleigh scattering. For more details of this updated model, see Section 2.

In the present paper, the last mentioned model, developed for the fovea, was applied to a number of parafoveal spots at increasing eccentricity. Our aim was to find out whether the parameters generated by the model were meaningful by comparing them to quantitative and qualitative literature data. We were mainly interested in the absorbers zeaxanthin, lutein, melanin, and blood, and in the directional properties of the photoreceptors, also known to change with eccentricity. ${ }^{20}$ At these peripheral locations, the retina is much thicker than at the fovea and, in particular, the nerve fiber layer may cause extra reflection. In addition, rod photoreceptors are interspersed with the cones. Spectral reflection from eccentric locations was recently reported, aimed at determining the retinal distribution profiles of zeaxanthin and lutein, the constituents of the macular pigment. ${ }^{21}$ The instrument used in that study lacked directional information though. In the present paper, we describe eccentric data obtained with the foveal reflection analyzer (FRA) (see Section 2), which incorporates the directional aspects.

\section{Methods}

\subsection{FRA Measurements}

The FRA was used for the measurement of the retinal reflection in a group of 23 young subjects with a mean age \pm SD of $24.3 \pm 2.7$ years. ${ }^{17,22}$ The FRA illuminates a 1.8-deg spot

$1083-3668 / 2010 / 15(6) / 065005 / 9 / \$ 25.00$ (C) 2010 SPIE 
centered on the fovea $(6.42 \log \mathrm{Td})$. Using a sensitive $\mathrm{CCD}$, the spectral and directional distribution of the reflection light is measured by a prism-based imaging spectrometer using only the light from an exit slit of $15 \times 1 \mathrm{~mm}$ over the natural pupil as input. Thus, the image on the CCD detector has in one dimension the directional information from the irradiance distribution over the slit-shaped exit pupil (0.1-mm resolution). In the other dimension, it contains the spectral information ranging from 400 to $950 \mathrm{~nm}$, as light from each point of the slit is decomposed by the prism. Only light from the central 1.5-deg foveal spot was used for detection. For a detailed description of the FRA, including a characterization of the light source, see van de Kraats and van Norren. ${ }^{17}$ Calculations showed that the light spot could be viewed safely for $15 \mathrm{~min}$ for one, nonoverlapping, retinal location. ${ }^{23}$ Because of the high measuring light levels, visual pigments were considered to be fully bleached; their absorption could therefore be neglected.

Before the experiment started, the nature of the experiment was explained to the subjects and written informed consent was obtained. The tenets of the Declaration of Helsinki were followed, and the local Medical Ethics Committee of the UMC Utrecht approved the research protocol. The pupil of one eye was dilated by topically instilling tropicamide $0.5 \%$ eye drops. Reflection was measured at the central fovea, along a line at 1,2 , 4 , and 8 deg eccentricities, and at the optic disk. For the foveal measurements, the subjects were asked to fixate at the center of the bright illumination spot. For the eccentric measurements, subjects were asked to fixate at one of the several small, red spots derived from a red light-emitting diode (LED). Images of the retina and the pupil plane were monitored continuously to assure stable fixation and as an aid during alignment to find the location in the pupil plane with the highest amplitude of the directional reflection (peak of the optical Stiles-Crawford effect) at 500-600 nm; at the same time minimizing the reflection near $400 \mathrm{~nm}$. A high reflection near $400 \mathrm{~nm}$ indicates unwanted corneal reflections. For the eccentric locations, a typical specular reflection from the ILM was often clearly visible at certain pupil positions during the alignment procedure. ${ }^{24}$ It was avoided by a small displacement of the instruments illumination beam in the pupil plane. The location of the optic disk was searched for by moving an external fixation LED for the fellow eye and simultaneously viewing the retinal image. The blood-vessel-rich area of the optic disk was avoided. After aligning the subject, one single measurement took $\sim 1 \mathrm{~s}$. Five measurements were taken at each retinal location, each time with reoptimized alignment.

\subsection{Reflection Model}

An optical reflection model (as described in detail earlier) was used for analyzing the measurement data. ${ }^{17}$ Basically, the complex reflection and absorption properties in the different tissue layers of the eye were reduced to four reflecting layers, separated by four layers with specific absorbers (see Fig. 1). Reflection was assumed to take place at the ILM $\left(R_{\mathrm{ILM}}\right)$, at the outer segment disks in the cone photoreceptors $\left(R_{\mathrm{disc}}\right)$, at the RPE $\left(R_{\mathrm{RPE}}\right)$, and in the choroidal space $\left(R_{\mathrm{chor}}\right)$. All reflections were assumed to be diffuse and spectrally neutral, except the reflection from the cones which was assumed to have $\lambda^{-2}$ behavior due to wave guiding processes and also to exhibit directional illumination detection

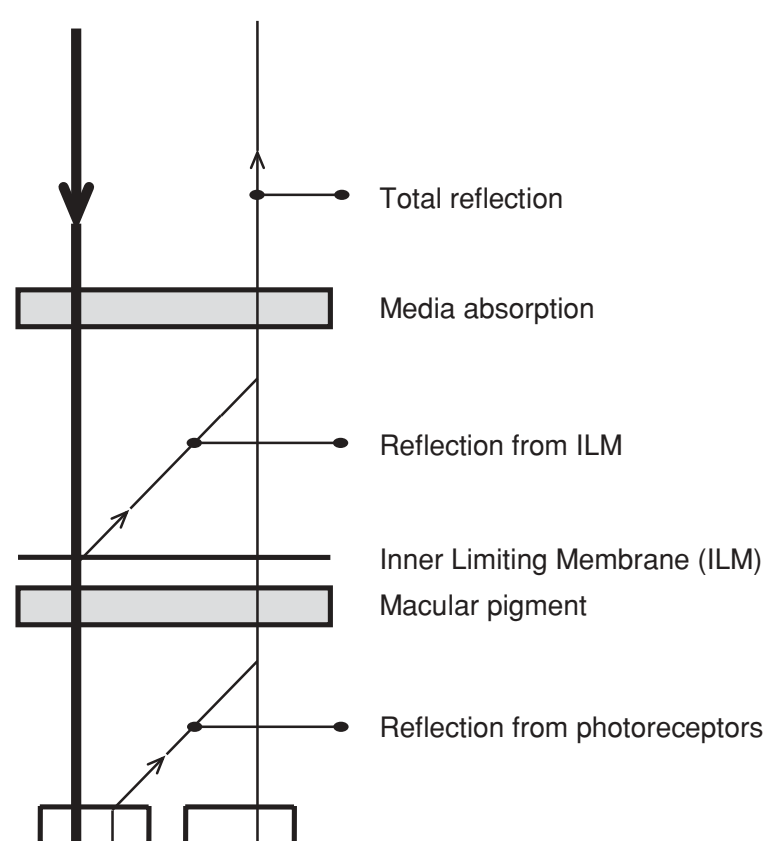

Photoreceptors

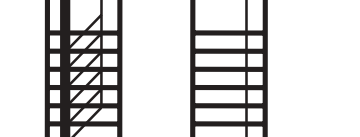

Photoceptors

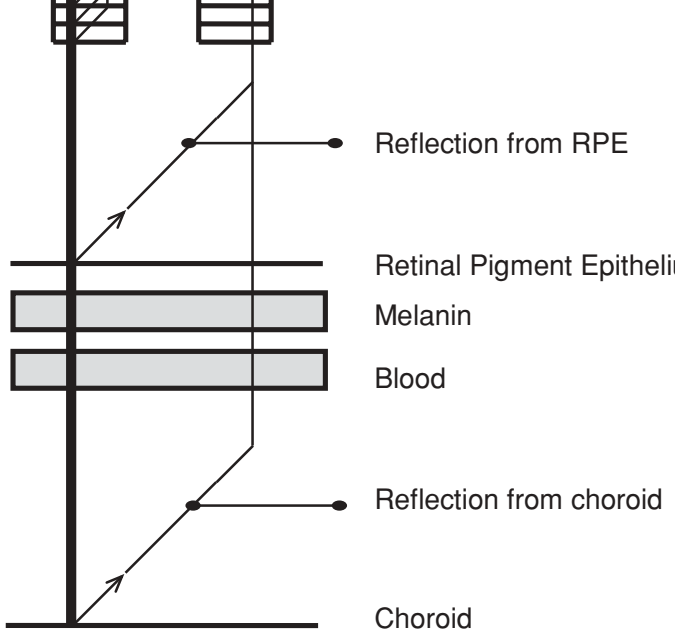

Fig. 1 Model of the reflection of the fovea. Light enters the eye at the left (thick line, illumination) and meets several layers of tissues in the anterior eye, receptor layer, and deeper layers posterior to the retina. Absorbing layers are shown as boxes and reflecting layers as horizontal lines. Reflection takes places at four layers and is symbolized by lines angled at $45 \mathrm{deg}$, continuing as the thin line at the right going upward to the detector. The only source of directional reflection is from photoreceptor disks, all others are nondirectional. For details see Section 2.

properties (discussed later). ${ }^{18,19}$ Rod receptors were assumed to be completely transparent and to have no directionality. ${ }^{25-28}$ Absorption was assumed to take place in the eye media, mainly the eye lens. Here, three components were distinguished. First, a component LY (which stands for lens young), with a kynureninelike spectral absorption that is abundantly present in the young lens (with parameter $d_{\mathrm{LY}}$ standing for the optical density at $400 \mathrm{~nm}) .{ }^{29}$ Second, a component LO (lens old) representing 
a component that becomes apparent with increasing age $\left(d_{\mathrm{LO}}, 400 \mathrm{~nm}\right)$, and third, RL (Raleigh losses), a component that stands for Rayleigh scatter losses $\left(d_{\mathrm{RL}}, 400 \mathrm{~nm}\right) .{ }^{29}$ Absorption in macular pigment $\left(d_{\mathrm{mp}}\right)$ was decomposed in zeaxanthin- and luteinlike spectral components with parameters for the optical densities at $460 \mathrm{~nm} d_{\text {zeax }}$ and $d_{\mathrm{lu}}$, and using lutein and zeaxanthin extinction spectra. ${ }^{21,30}$ In the deeper layers, posterior to the photoreceptors, the amount of absorption in melanin and blood was described with the optical density parameter $d_{\text {mel }}$ (at $500 \mathrm{~nm}$ ) and $h_{\text {blood }}$ (layer thickness in millimeters), whereby melanin is located in both the RPE and the choroidal space. A fundamental issue is the distinction between the directional reflection from the cone outer-segment disks and the nondirectional reflection from the other layers. As stated in Section 1, the optical model takes advantage of this to better discriminate between parameters. The originally measured spectral data were separated (see Fig. 2) in a directional and a nondirectional data component. This was useful for comparison to the directional and a nondirectional components produced by the optical model itself (both shown in Fig. 3). The directionality (peakedness) of the Gaussian is characterized by the parameter $\rho$ (per millimeters squared). The Levenberg-Marquard routine was used to fit the data with the model by minimizing $\chi^{2}$ values. ${ }^{31} \mathrm{~Pa}$ rameters for the absorbing and reflecting layers were either allowed to vary simultaneously, or they were fixed to a stated value.

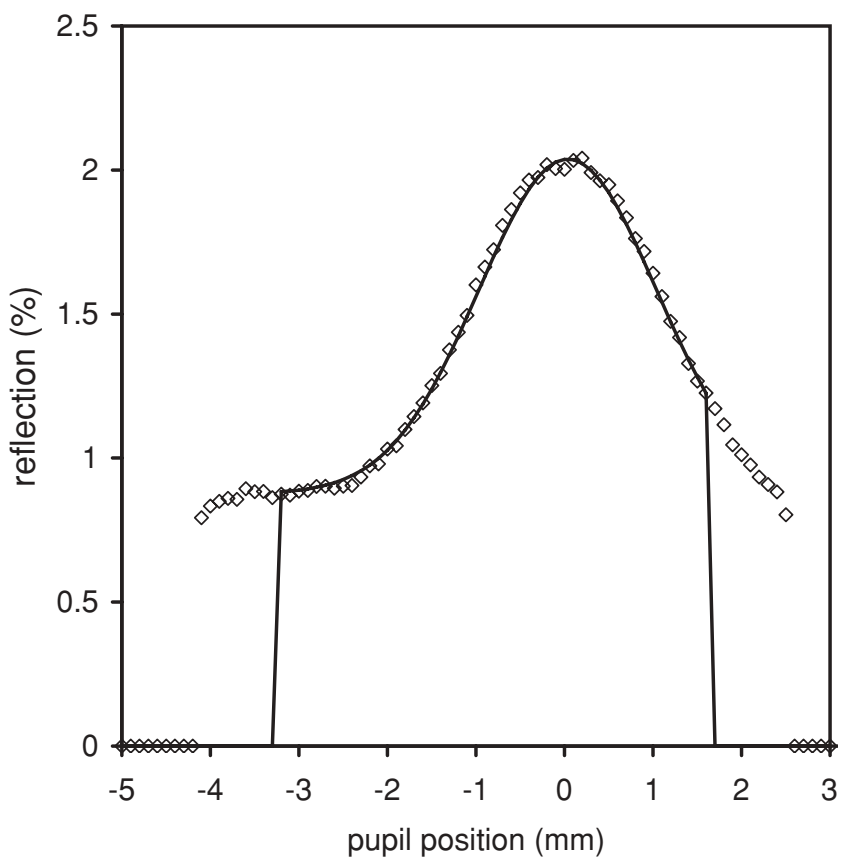

Fig. 2 Example of a single measurement of the irradiance distribution at $540 \mathrm{~nm}$ in the pupil plane (data points). A Gaussian model curve was fitted to the data keeping a distance of at least $1 \mathrm{~mm}$ to the edges of the pupil. The amplitude of the Gaussian curve $(\sim 1.2 \%)$ is used as a measure for the amount of the directional reflection. The height of the pedestal $(\sim 0.8 \%)$ is a measure for the nondirectional reflection. The zero position on the pupil axes is linked to the peak of the function due to the alignment procedure and is generally near the center of the pupil.
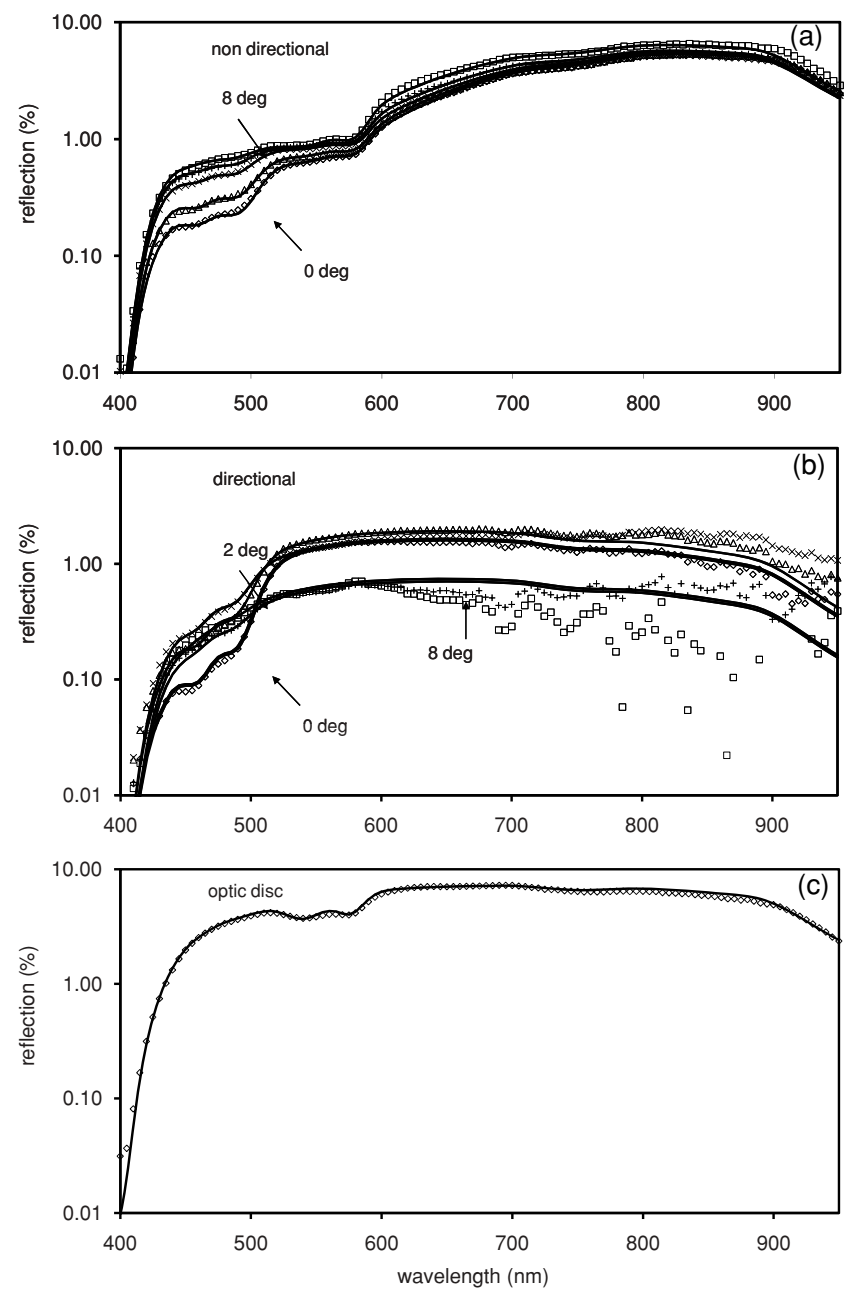

Fig. 3 Mean reflection spectra for the 23 subjects (data points); continuous model curves were fitted to the data. (a) Nondirectional reflection at 0 (diamonds), 1 (triangles), 2 (crosses), 4 (plusses), and 8-deg eccentricity (boxes). (b) Same as (a) but for the directional reflection at the same five locations. Note that the model curves were not directly fitted to the data displayed here, but to the complete two-dimensional measured dataset (see Section 2). (c) Nondirectional reflection from the optic disk. A low-amplitude directional component is not presented because it did not show the typical cone imprint and therefore carried no extra spectral information.

\section{Results}

\subsection{FRA Measurements}

Figure 2 shows an example of the irradiance distribution measured in the pupil plane for $540 \mathrm{~nm}$. The data were decomposed by the model analysis in a Gaussian with an amplitude that stands for the directional component of the retinal reflection and a pedestal that stands for the nondirectional reflection. Profiles at all wavelengths from 400 to $950 \mathrm{~nm}$ become available in a single measurement (not shown).

The mean nondirectional and directional spectral traces of the 23 subjects for five retinal locations from zero to $8 \mathrm{deg}$ are shown in Figs. 3(a) and 3(b). The continuous model curves will be described later. The data points of the nondirectional spectra in Fig. 3(a) shows the typical increase in reflection from 400 to $430 \mathrm{~nm}$, caused by the declining absorption in the lens. The steplike increase in reflection near $500 \mathrm{~nm}$ is caused by the steep 


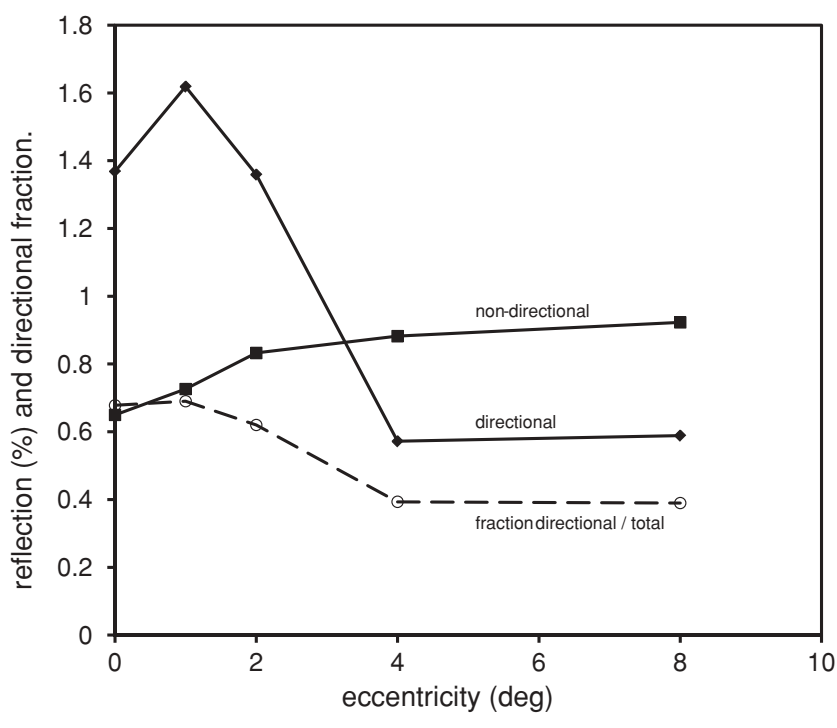

Fig. 4 Measured amplitudes at $550 \mathrm{~nm}$ of the directional reflection (diamonds) and the nondirectional reflection (filled squares) as a function of eccentricity. The fraction of the directional reflection to the total reflection is also shown (dashed line).

decrease in absorption of the macular pigment. For the higher eccentricities, the amplitude of this step diminishes. Above $580 \mathrm{~nm}$, reflection further increases because of the strong decline in the absorption of blood. The main absorber remaining in that part of the spectrum is melanin, which also shows a decline in absorption with increasing wavelength thus further increasing reflectance at longer wavelengths. At the longest wavelengths, reflection starts declining because the absorption of water is no longer negligible. The directional spectra in Fig. 3(b) are quite similarly shaped as those of the nondirectional reflection from 400 to $580 \mathrm{~nm}$. However, above $580 \mathrm{~nm}$, they lack the typi- cal increase because of the decreasing absorption in blood and melanin (cf. Section 1). Above $580 \mathrm{~nm}$, the declining fraction of the directional reflection compared to the total reflection, caused rather noisy data.

Figure 3(c) shows the reflection spectrum of the optic disk, with the expected absence of the spectral fingerprints of macular pigment and melanin. The directional spectrum proved to be just a low-amplitude version of the nondirectional one, caused by a slightly ramping irradiance distribution over the pupil (not shown).

Figure 4 shows the mean amplitudes of the directional and nondirectional component as a function of eccentricity for a single wavelength of $550 \mathrm{~nm}$. For the directional reflection at wavelengths from 550 to $700 \mathrm{~nm}$, the curves had a similar shape; below $550 \mathrm{~nm}$, the absorption in macular pigment and in the eye media caused a decrease in amplitude (not shown). The nondirectional shape at other wavelengths was more complex in amplitude due to additional influence of absorption in melanin and blood (not shown).

\subsection{Parameters Derived from Reflection Model}

The optical reflection model was applied to the data. The mean spectra generated by the model are shown as continuous lines in Fig. 3. They generally show a very good fit to the data. An exception occurs when there is excessive noise in the data points of the directional amplitude at the longer wavelengths. In particular, at the higher eccentricities these directional data values were a relatively low fraction $(\sim 10 \%)$ of the total reflection. It should be realized that the less optimal model fitting in these cases is therefore mainly caused by minimizing the $\chi^{2}$ in the two-dimensional data space (see Section 2) and not to the single traces of the nondirectional and directional components as shown in Fig. 3. In order to prevent interaction with the retinal model parameters, for these young subjects

Table 1 Optical model parameters as a function of retinal location. Bold values were fixed in the fitting process. Values of the standard error of the mean are in parenthesis.

\begin{tabular}{|c|c|c|c|c|c|c|}
\hline Location (deg) & 0 & 1 & 2 & 4 & 8 & Optic disk \\
\hline Zeaxanthin (d.u. at $460 \mathrm{~nm}$ ) & $0.270(0.022)$ & $0.159(0.012)$ & $0.061(0.006)$ & $0.022(0.005)$ & $0.008(0.003)$ & $0.001(0.001)$ \\
\hline Lutein (d.u. at $460 \mathrm{~nm}$ ) & $0.188(0.015)$ & $0.135(0.013)$ & $0.108(0.010)$ & $0.063(0.007)$ & $0.040(0.007)$ & $0.006(0.000)$ \\
\hline Macular pigment (d.u. at $460 \mathrm{~nm}$ ) & $0.458(0.031)$ & $0.294(0.021)$ & $0.170(0.014)$ & $0.085(0.009)$ & $0.048(0.008)$ & $0.007(0.001)$ \\
\hline Zeaxanthine fraction & $0.586(0.021)$ & $0.550(0.026)$ & $0.375(0.032)$ & $0.271(0.051)$ & $0.145(0.045)$ & 0.145 \\
\hline Melanin (d.u. at $500 \mathrm{~nm}$ ) & $1.381(0.065)$ & $1.371(0.068)$ & $1.294(0.064)$ & $1.188(0.056)$ & $1.070(0.056)$ & $0.018(0.009)$ \\
\hline Blood (mm) & $0.071(0.007)$ & $0.076(0.007)$ & $0.084(0.007)$ & $0.082(0.007)$ & $0.077(0.008)$ & $0.051(0.003)$ \\
\hline ILM reflection (\%) & $0.112(0.013)$ & $0.118(0.019)$ & $0.190(0.028)$ & $0.140(0.030)$ & $0.198(0.043)$ & $0.000(0.000)$ \\
\hline Disk refl. (\%) & $3.827(0.201)$ & $4.518(0.215)$ & $3.810(0.169)$ & $1.665(0.113)$ & $1.751(0.135)$ & 0.000 \\
\hline$\rho\left(\mathrm{mm}^{-2}\right)$ & $0.151(0.010)$ & $0.180(0.008)$ & $0.193(0.008)$ & $0.092(0.011)$ & $0.071(0.005)$ & \\
\hline RPE reflection (\%) & $0.527(0.032)$ & $0.636(0.039)$ & $0.672(0.043)$ & $0.724(0.050)$ & $0.655(0.062)$ & $2.951(0.223)$ \\
\hline Choroid refl. (\%) & $7.663(0.285)$ & $7.844(0.260)$ & $7.658(0.290)$ & 7.793 (0.277) & $8.593(0.346)$ & $4.848(0.345)$ \\
\hline
\end{tabular}



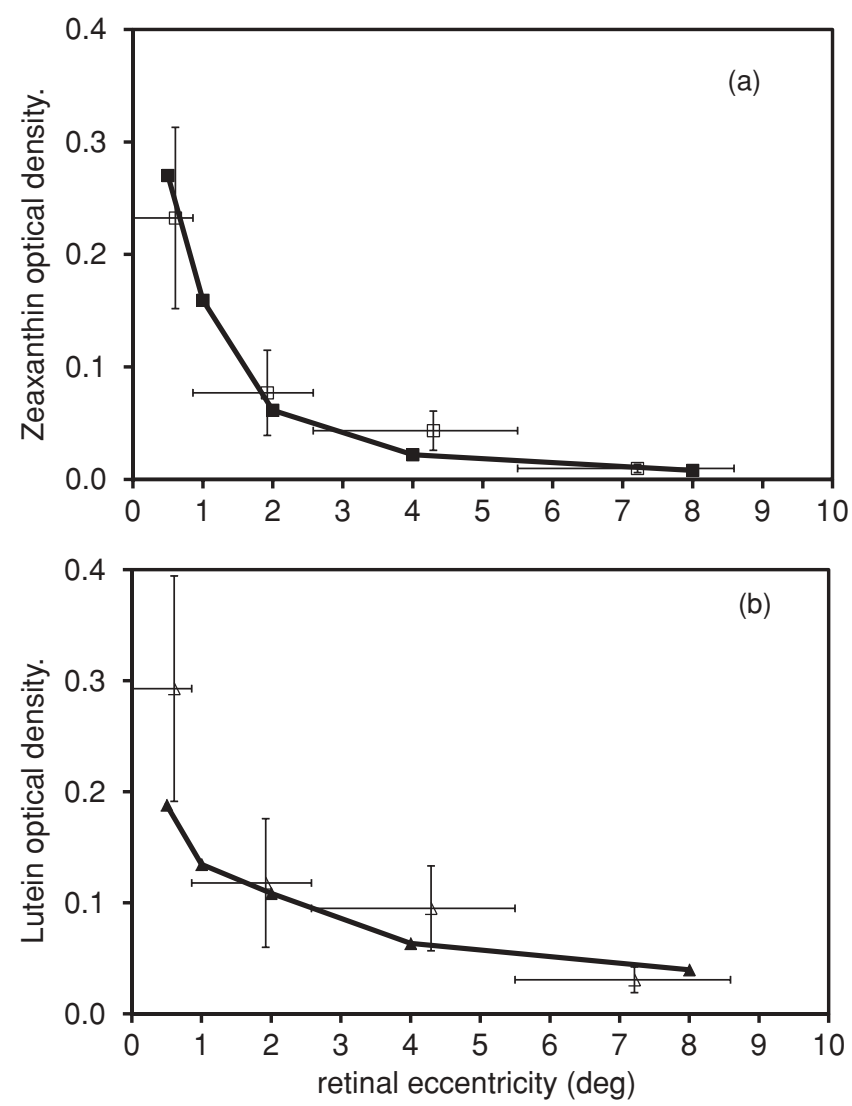

Fig. 5 (a) Spatial profiles of zeaxanthin and (b) lutein showing the data points (filled large squares) connected by a thick solid line. For comparison, HPLC data from Bone et al. ${ }^{32}$ derived from donor retina is shown (squares). Error bars to the Bone data show the standard deviation (vertical) and the region over which the data was averaged (horizontal).

(mean age $24.3 \pm 2.7$ years) with their transparent media, we fixed the components for the density of the media at the mean values according to an aging-eye media transmission formula, ${ }^{29}$ that is $d_{\mathrm{LY}}=1.13$ and $d_{\mathrm{LO}}=0.09$. In preanalyses of the data for the locations 0,1 , and $2 \mathrm{deg}$, the Rayleigh scatter parameter $d_{\mathrm{RL}}$ was at first allowed to vary. These locations showed a high directional reflection, enhancing the detection of anterior absorbers. A resulting mean of 0.77 (with a low SD of 0.008) was used to set $d_{\mathrm{RL}}$ as a fixed parameter value in the final model fit calculations for all locations, as we assumed that the angles of the light beams through the media changed only slightly. The remaining free parameters of the optical model as a function of eccentricity are presented in Table 1, and depicted in Figs. 5-7 (absorbers), Fig. 8 (reflectors), and Fig. 9 (directionality). Their correspondence with literature data is discussed in Section 3.3.

For the analysis of the measurements from the optic disk, devoid of cones [Fig. 3(c)], the low-directional component was fixed at zero. The zeaxanthin fraction was fixed to the value derived from the analysis at the most eccentric location $(8 \mathrm{deg})$. Results of the other model parameters are in Table 1.

\subsection{Comparison of Parameters with Literature Data}

\subsubsection{Macular pigment}

The retinal distribution data on $d_{\text {lut }}$ and $d_{\text {zeax }}$ show a qualitative correspondence with HPLC mass data from Bone et al. ${ }^{32}$
(Fig. 5) and other literature data ${ }^{33-35}$ (not shown) in the sense that $Z$ falls off more steeply than $L$. The present data with the FRA are similar to those obtained with another instrument (the macular pigment reflectometer) on the same subjects. ${ }^{21}$ The profile of the macular pigment $\left(d_{\mathrm{mp}}\right)$ as the sum of the optical densities of lutein and zeaxanthin is compared to literature data in Fig. 6. A high correspondence is seen with earlier data from our laboratory obtained from 53 subjects using scanning laser ophthalmoscopic (SLO) autofluorescence images, ${ }^{36}$ and with recent data of 24 subjects with a comparable mean age of 24.8 years obtained by a reflectometric method (at 460 and $560 \mathrm{~nm}$ ) from Chen et al. ${ }^{37}$

\subsubsection{Melanin}

Both RPE and choroidal melanin have a peak in the central fovea. ${ }^{38-40}$ However, detailed literature data of $d_{\text {mel }}$ versus retinal eccentricity is lacking. The current results are shown in Fig. 7.

\subsubsection{Blood}

Blood layer thickness was relatively stable at the different eccentricities, with somewhat lower values in the central fovea (Fig. 7). Like melanin, detailed literature data on the thickness of the blood layer versus eccentricity is lacking.

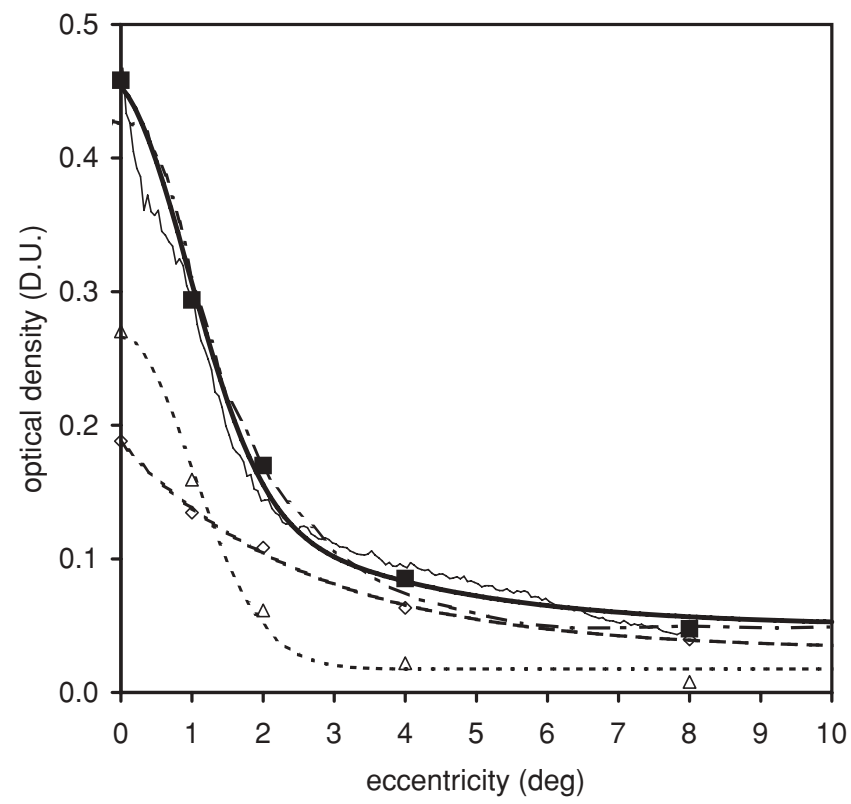

Fig. 6 Spatial profile of macular pigment showing the data points (filled large squares), and the model (thick solid line). The model consists of the sum of lutein (diamonds), modeled by an exponential (dashed line) and zeaxanthin (triangles) modeled by a Gaussian (dotted line). For comparison of the shape, detailed SLO autofluorescence results from Berendschot and van Norren ${ }^{36}$ (thin line), and from Chen et al. ${ }^{37}$ (dotted-dashed line) were shown. These data were originally forced to zero at the reference point of $8 \mathrm{deg}$. The present derived $d_{\mathrm{mp}}$ value at $8 \mathrm{deg}(0.048)$ was therefore added to both curves; in addition, the Chen data was multiplied by a factor 1.7 . 


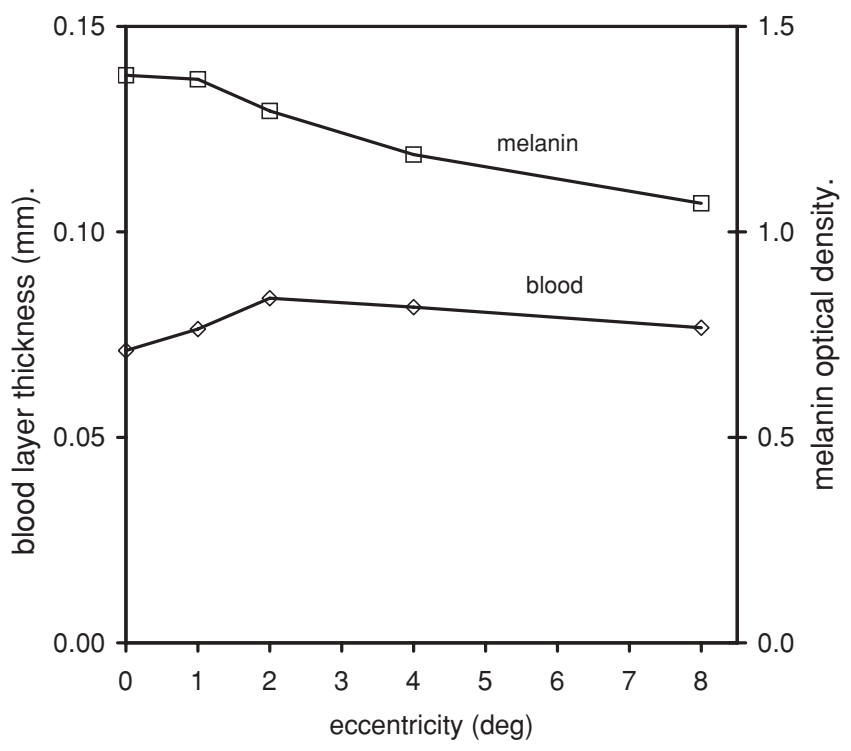

Fig. 7 Parameter values for blood and melanin in the reflection model as a function of eccentricity. The data points are connected for clarity. The blood data (diamonds) are in millimeter blood-layer thickness (lefthand scale). Melanin data (squares) are in optical density units (righthand scale).

\subsubsection{Retinal reflectors}

To our knowledge literature data for the amplitudes of the directional or nondirectional reflectors versus eccentricity are not available. The current results are in Fig. 8.

\subsubsection{Directionality}

The current data can be described by the sum of a Gaussian $0.144+0.088^{*} 10^{-\left[0.4^{*}\left(\mathrm{deg}^{+}-1.7\right]^{2}\right.}$ and an exponential

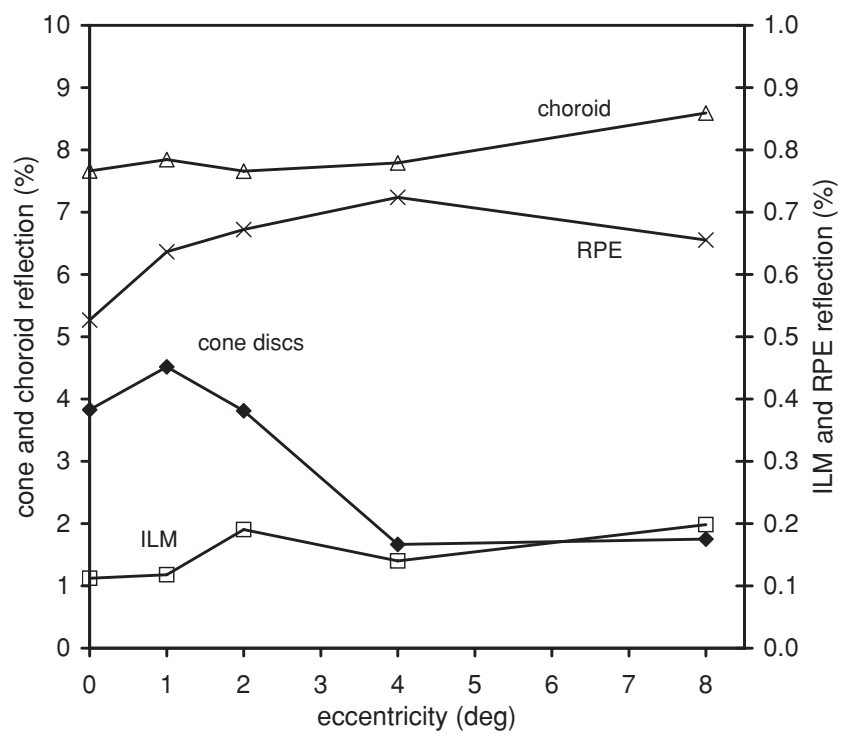

Fig. 8 Parameter values for the reflecting layers in the reflection model as a function of eccentricity. The data points are connected for clarity. Shown are the reflection in percent from the ILM (squares), the cone discs (filled diamonds), the RPE (crosses), and the reflection from the choroidal space (triangles). The much smaller ILM and RPE reflections refer to the right-hand scale.

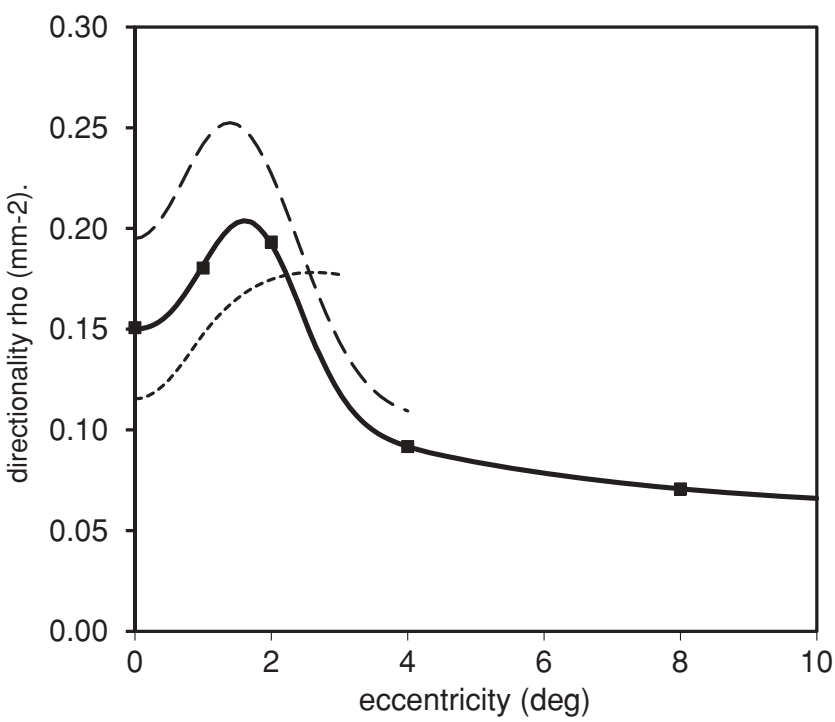

Fig. 9 Directionality of the cones (squares) as a function of eccentricity compared to other fundus reflectometric data from Burns et al. ${ }^{14}$ (dots), and from de Lint et al. ${ }^{45}$ (dashes). A model curve, being the sum of a Gaussian and an exponential (solid line), was fitted through the current data.

$-0.0858^{*}\left[1-\exp \left(-0.2405^{*} \mathrm{deg}\right)\right]$, showing that the directionality of the reflection from the slender cone outer segments at the central fovea was low $(0.163)$ compared to the more robust cones at 1 and $2 \operatorname{deg}(0.193$ and 0.203 , respectively), as generally found in the literature. ${ }^{41-44}$ Data for reflection techniques were available from two sources (Fig. 9). ${ }^{14,45}$ Individual shapes for $\rho$ versus eccentricity were first convolved over the 1.5-deg detection area of the FRA. Data from Burns et al. ${ }^{14}$ is the mean over four quadrants and does not show a clear downward tail; they end at 3 deg eccentricity. Data from de Lint et al., ${ }^{45}$ available up to $4 \mathrm{deg}$, look similar to the current data. Note that a direct comparison is not possible because of differences in entrance and exit pupil configuration.

\subsubsection{Density of the eye media}

Literature data for the mean age of 24 years were derived from a recent eye media transmission formula based on an extensive review. ${ }^{29}$ For the reflection 0-8 deg, only the parameter $d_{\mathrm{RL}}$ was allowed to vary (in a preanalysis), resulting in a higher density (0.77) than derived from the formula (0.47).

\section{Discussion}

We investigated the application of a foveal reflection model to more peripheral retinal sites. In general, results were obtained that corresponded well to the (limited) literature data available. Thus, extra retinal layers, rods, and changes in the directionality of cones seem no obstacle for the application of the model. The results are discussed in more detail in Sections 4.1-4.7.

\subsection{Macular Pigment}

The value for $d_{\mathrm{mp}}$ at the central fovea, for which many sources exists, shows large inter-individual variations in the literature. Also, the type of technique used and the size of the retinal field 
sampled make a direct comparison to values from the literature difficult.

Delori et al. ${ }^{46}$ compared different methods to obtain $d_{\mathrm{mp}}$ in a group of 159 subjects using a diameter of the retinal field of $2 \mathrm{deg}$. The reflection method yielded a $d_{\mathrm{mp}}$ of 0.23 while $d_{\mathrm{mp}}$ was found to be 0.48 using the autofluorescence of lipofuscin. A $d_{\mathrm{mp}}$ value of 0.37 was found with the psychophysical method of flicker photometry. The present $d_{\mathrm{mp}}$ value for the central fovea of 0.46 was somewhat higher compared to an earlier result of 0.39 obtained from another group measured with the same instrument. ${ }^{17}$ A central $d_{\text {mp }}$ value of 0.63 was found with another reflectometer device using a smaller field size of 1 deg. ${ }^{47}$ For an older-aged group (mean age $68 \pm 5$ ) Berendschot et al. ${ }^{48}$ found a central $d_{\mathrm{mp}}$ of 0.33 , but they used a 2.3-deg field. More examples of the psychophysical method using flicker photometry for determining a central $d_{\mathrm{mp}}$ delivered 0.22 for a 1 -deg field ${ }^{49}$ and 0.63 for a 1.6 -deg field. ${ }^{50}$ As an aid to future model work, the current lutein data versus eccentricity are well described by an exponential $(0.032$ $\left.+0.155^{*} 10^{-0.166^{*} \mathrm{deg}}\right)$, and zeaxanthin with a steeper exponential $\left(0.003+0.272 * 10^{-0.284 * \mathrm{deg} * \mathrm{deg}}\right)$.

At $8 \mathrm{deg}$, the value for $d_{\mathrm{mp}}$ was still measurable with 0.048 and with 0.085 at $4 \mathrm{deg}$. Even without any model analysis the small inflection near $500 \mathrm{~nm}$ in the raw data of Fig. 3(a) is indicative for absorption by the macular pigment. This finding is important for many (psychophysical) methods, which use an eccentric reference point and often rely on the assumption that $d_{\mathrm{mp}}$ at that location is negligible. ${ }^{36,51,52}$ Recently, van der Veen et al. discussed this issue in detail when comparing data obtained by an optical and a psychophysical method in the same subjects. ${ }^{35}$ As a check, we also measured reflection at the optic disk; we found a mean $d_{\text {mp }}$ of only 0.007 . This low $d_{\text {mp }}$ value could, in principle, also originate from macular pigment present in the eye lens. ${ }^{53-55}$

\subsection{Melanin}

The modeling of the choroid is too simplified to provide meaningful parameters in absolute sense. Recent high-resolution autofluorescence images at $787 \mathrm{~nm}$ were assumed to originate from melanin. ${ }^{56}$ These images show Gaussian-like profiles similar to the present data, up to $4 \mathrm{deg}$. Again, for future modeling purposes an almost perfect description of the melanin distribution over the full eccentricity range from this paper is provided by the Gaussian equation $1.066+0.315^{*} 10^{-0.027 * \operatorname{deg} * \operatorname{deg}}$ (not shown).

\subsection{Blood}

The increased melanin absorption in the central fovea might have reduced the blood layer thickness results at the central fovea, due to the optical model being not advanced enough in the deeper retinal layers. ${ }^{17}$ Delori and Pflibsen ${ }^{8}$ used KubelkaMunk modeling of the deeper layers to tackle this problem, but making a direct comparison to the currently derived values in absolute sense is difficult. ${ }^{8}$ As in the present data, they found a lower value in the fovea $(0.168 \mathrm{~mm})$ than at $2.5 \mathrm{deg}$ $(0.182 \mathrm{~mm})$.

\subsection{Retinal Reflectors}

The choroidal reflection showed a slight increase with eccentricity. The reflection from the ILM was low at the central fovea, where the nerve fiber layer is very thin. The ILM reflection increased with eccentricity, in line with the increase in thickness of the nerve fiber layer. We assumed reflection from the ILM to be spectrally neutral. This might be an oversimplification outside the fovea because the nerve fiber layer reflectance was found to rise as $\lambda^{-n}$ from 560 to $460 \mathrm{~nm}$, where $n=2.5-3 .{ }^{57,58}$ For wavelengths beyond $560 \mathrm{~nm}$, only minor changes were reported.

The reflection from the RPE varied only marginally over the eccentricities used. Rods have virtually no directionality; ${ }^{25-28}$ thus, light reflected by the rods adds to the nondirectional RPE reflection. Rods were transparent because the high light levels used bleached their rhodopsin content. Light transmitted by the rods is therefore added to the reflection from the choroid.

The amplitude of the directional component as a function of eccentricity resembles that of the directionality, $\rho$, as a function of eccentricity (see Figs. 4 and 9). It can be assumed that the light received by an isolated cone is linear with the antenna gain proportional with $\rho$. Similarly, this holds for the light transmitted by a cone on the way out. As a result, we suppose the amplitude of the directional reflection to behave linearly with $\rho^{2}$. However, when cones are closely packed, the relation at entry does no longer hold because of mutual competition for the available light. Thus, for the current data, the shape of the profile of the reflected light in the pupil plane is only affected by the directionality when transmitting because the receiving light is always entering at the peak of the SCE. Therefore, we expect a linear relation between $\rho$ and the amplitude of the directional reflection.

In the model, it is hypothesized that this reflection is proportional with the number of disks inside the cone outer segments. ${ }^{13}$ Recent high-resolution OCT images rather indicate discrete reflections at both ends of the outer segments. ${ }^{59}$ With $842-\mathrm{nm}$ light, these end reflections exhibited directional properties. ${ }^{15}$ Reflections from the disks in the outer segment however are not discernable with the current resolution. For this paper, these differences in interpretation are of no importance.

\subsection{Directionality}

The directionality as function of eccentricity showed similarity in shape to reflectometric literature data up to 4 deg. ${ }^{14,45} \mathrm{Re}-$ cently, Gao et al. extracted directional sensitivities from ShackHartmann wavefront sensor measurements. ${ }^{60}$ After accounting for differences in wavelength and configuration of entrance and exit pupils, their results are consistent with data obtained with conventional reflectometers. The directionality obtained with optical techniques seems to be related to reflections near the posterior tip of the photoreceptor outer segments. ${ }^{15}$ It has been suggested that the directionality is connected to the shape of the retinal receptors. ${ }^{41}$ Modeling indeed showed that an elongated central cone will have a lower directionality than a shorter and broader eccentric cone. ${ }^{61}$ The rise from 0 to 2 deg supports this theory. The decrease from 2 deg onward is not seen in psychophysical measurements. However, psychophysical tests are generally designed such that only cones are stimulated, and cone shape changes little beyond 2 deg eccentricity. Optical test results, on the contrary, are due to a combination of cone and rod reflectance, with rods having no or only minimal directional behavior. $^{25-28}$ It seems likely that the observed decrease in the 
directionality from 2 deg onward is due to the increasing rod to cone ratio.

\subsection{Density of Eye Media}

The retinal reflection from 0 to $8 \mathrm{deg}$ yielded a good fit to the data when the Rayleigh scatter value $d_{\mathrm{RL}}$ was set to the mean of a preanalysis (0.77). Using the mean literature value 0.47 from the eye media formula, the mean $\chi^{2}$, as a figure of merit from the fitting, became $\sim 20 \%$ higher. Also, as judged by eye, the model curves did not follow the data very well. The high value 0.77 is probably not caused by scatter losses in the nerve fiber layer, because the value for the fovea was similar to the values at 1 and $2 \mathrm{deg}$; the SD from the values at these three locations was only 0.008 . We speculate that the assumption about the shape of the spectral reflection from the cones by the $\lambda^{-2}$ wave guiding behavior is not fully correct and accounts for the difference between 0.77 and 0.47 . Alternatively, the value for $d_{\mathrm{RL}}$ from the eye media formula could also be subject to error. This formula was partly based on mean data from donor lenses, which can have deteriorated tissue, and on psychophysical experiments, which perhaps contain scatter losses in the retina itself.

\subsection{Optic Disk Reflection}

The optical density of only 0.007 for macular pigment was indicative for its absence at the optic disk location. This low $d_{\mathrm{mp}}$ value found could, in principle, also originate from macular pigments present in the eye lens. ${ }^{53-55}$ Melanin is also thought to be absent at the disk. The optical density of 0.018 might be accounted for by scattered light originating from a region at which melanin is present. A blood layer thickness of $51 \mu \mathrm{m}$ might be due to occasional (inadvertent) positioning of the spot at a blood vessel, due to less stable fixation with the other eye. The largest part of the total reflection from the optic disk was allotted to the parameter for the choroid reflection (4.85\%). The reflections found for the (virtual parameters) ILM and the RPE can be added to $2.95 \%$. This simplifies the spectral reflection model of the optic disk itself to two neutral reflectors with absorption by melanin and blood in between. The low value of $d_{\mathrm{RL}}$ might be caused by the reflectors of the optic disk model deviating from neutral (pure white). At least some scattering losses are expected in the trabecular meshwork and in the nerve fibers at that location.

\section{Conclusion}

A recent model that was developed to explain directional and nondirectional spectral reflection data of the fovea seems also capable of handling data from eccentric retinal locations. The model generally shows a good fit to the data, and model parameters as a function of eccentricity show a behavior that corresponds to the (sometimes scarce) literature data.

\section{References}

1. G. S. Brindley and E. N. Willmer, "The reflexion of light from the macular and peripheral fundus oculi in man," J. Physiol. 116, 350-356 (1952).
2. H. Ripps and R. A. Weale, "Analysis of foveal densitometry," Nature 205, 552-556 (1965)

3. W. A. H. Rushton, "Straylight and the measurement of mixed pigments in the retina," J. Physiol. 176, 46-55 (1965).

4. M. Alpern and F. W. Campbell, "The spectral sensitivity of the consensual light reflex," J. Physiol. 164, 478-507 (1962).

5. H. Ripps and A. G. Snapper, "Computer analysis of photochemical changes in the human retina," Comput. Biol. Med. 4, 107-122 (1974).

6. R. A. Weale, "Further studies of photo-chemical reactions in living human eyes," Vis. Res. 1, 354-378 (1962).

7. D. van Norren and L. F. Tiemeijer, "Spectral reflectance of the human eye," Vis. Res. 26, 313-320 (1986).

8. F. C. Delori and K. P. Pflibsen, "Spectral reflectance of the human ocular fundus," Appl. Opt. 28, 1061-1077 (1989).

9. M. Hammer, A. Roggan, D. Schweitzer, and G. Muller, "Optical properties of ocular fundus tissues - an in vitro study using the doubleintegrating-sphere technique and inverse Monte Carlo simulation," Phys. Med. Biol. 40, 963-978 (1995).

10. M. Hammer and D. Schweitzer, "Quantitative reflection spectroscopy at the human ocular fundus," Phys. Med. Biol. 47, 179-191 (2002).

11. T. T. J. M. Berendschot, P. J. de Lint, and D. Norren, "Fundus reflectance-historical and present ideas," Prog. Retin. Eye Res. 22, 171200 (2003).

12. G. J. van Blokland and D. van Norren, "Intensity and polarization of light scattered at small angles from the human fovea," Vis. Res. 26, 485-494 (1986).

13. J. van de Kraats, T. T. J. M. Berendschot, and D. van Norren, "The pathways of light measured in fundus reflectometry," Vis. Res. 36, 22292247 (1996).

14. S. A. Burns, S. Wu, J. C. He, and A. E. Elsner, "Variations in photoreceptor directionally across the central retina," J. Opt. Soc. Am. A 14, 2033-2040 (1997).

15. W. Gao, B. Cense, Y. Zhang, R. S. Jonnal, D. T. Miller, "Measuring retinal contributions to the optical Stiles-Crawford effect with optical coherence tomography," Opt. Express 16, 6486-6501 (2008).

16. J. Krauskopf, "Some experiments with a photoelectric ophthalmoscope," in Excerpta Medica Intl. Congress Series, Proc. of Performance of the Eye at Low Luminances, Vol 125, pp. 171-181, M. A. Bouman and J. J. Vos Eds., Delft, 1965.

17. J. van de Kraats and D. van Norren, "Directional and non-directional spectral reflection from the human fovea," J. Biomed. Opt. 13, 024010 (2008).

18. S. Marcos, S. A. Burns, and J. C. He, "Model for cone directionality reflectometric measurements based on scattering," J. Opt. Soc. Am. A 15, 2012-2022 (1998).

19. S. Marcos and S. A. Burns, "Cone spacing and waveguide properties from cone directionality measurements," J. Opt. Soc. Am. A 16, 995 1004 (1999).

20. C. A. Curcio, K. R. Sloan, R. E. Kalina, and A. E. Hendrickson, "Human photoreceptor topography," J. Comp. Neurol. 292, 497-523 (1990).

21. J. van de Kraats, M. J. Kanis, S. W. Genders, and D. van Norren, "Lutein and zeaxanthin measured separately in the living human retina with fundus reflectometry," Invest. Ophthalmol. Vis. Sci. 49, 5568-5573 (2008).

22. N. P. A. Zagers, J. van de Kraats, T. T. J. M. Berendschot, and D. van Norren, "Simultaneous measurement of foveal spectral reflectance and cone- photoreceptor directionality," Appl. Opt. 41, 4686-4696 (2002).

23. "Guidelines on limits of exposure to broad-band incoherent optical radiation ( 0.38 to 3 microM); International Commission on Non-Ionizing Radiation Protection," Health Phys. 73, 539-554 (1997).

24. J. M. Gorrand and F. C. Delori, "Reflectance and curvature of the inner limiting membrane at the foveola," J. Opt. Soc. Am. A 16, 1229-1237 (1999).

25. J. A. J. van Loo and J. M. Enoch, "The scotopic Stiles-Crawford effect," Vis. Res. 15, 1005-1009 (1975).

26. F. Flamant and W. S. Stiles, "The directional and spectral sensitivities of the retinal rods to adapting fields of different wavelengths," J. Physiol. (London) 187, 187-202 (1948).

27. G. Westheimer, "Directional sensitivity of the retina: 75 years of StilesCrawford effect," Proc. Biol. Sci. 275, 2777-2786 (2008). 
28. B. H. Crawford, "The luminous efficiency of light entering the eye pupil at different points and its relation to brightness threshold measurements," Proc. R. Soc. London Ser. B 124, 81-96 (1937).

29. J. van de Kraats and D. van Norren, "Optical density of the aging human ocular media in the visible and the UV," J. Opt. Soc. Am. A 24, 1842-1857 (2007).

30. G. J. Handelman, D. M. Snodderly, N. I. Krinsky, M. D. Russett, A. J. Adler, "Biological control of primate macular pigment. Biochemical and densitometric studies," Invest. Ophthalmol. Vis. Sci. 32, 257-267 (1991).

31. W. H. Press, B. P. Flannery, S. A. Teukolsky, and W. T. Vetterling, Numerical Recipes in Pascal: The Art of Scientific Computing, Cambridge University Press, Cambridge, England (1989).

32. R. A. Bone, J. T. Landrum, L. Fernandez, and S. L. Tarsis, "Analysis of the macular pigment by HPLC: retinal distribution and age study," Invest. Ophthalmol. Vis. Sci. 29, 843-849 (1988).

33. D. M. Snodderly, J. D. Auran, and F. C. Delori, "The macular pigment. II. spatial distribution in primate retinas," Invest. Ophthalmol. Vis. Sci. 25, 674-685 (1984).

34. D. M. Snodderly, G. J. Handelman, and A. J. Adler, "Distribution of individual macular pigment carotenoids in central retina of macaque and squirrel monkeys," Invest. Ophthalmol. Vis. Sci. 32, 268-279 (1991).

35. R. L. P. van der Veen, T. T. J. M. Berendschot, M. Makridaki, F. Hendrikse, D. Carden, and I. J. Murray, "Correspondence between retinal reflectometry and a flicker-based technique in the measurement of macular pigment spatial profiles," J. Biomed. Opt. 14, 064046 (2009).

36. T. T. J. M. Berendschot and D. van Norren, "Macular pigment shows ringlike structures," Invest. Ophthalmol. Vis. Sci. 47, 709-714 (2006).

37. S. F. Chen, Chang, Y., J. C. Wu, "The spatial distribution of macular pigment in humans," Curr. Eye Res. 23, 422-434 (2001).

38. V. P. Gabel, R. Birngruber, and F. Hillenkamp, "Visible and near infrared light absorption in pigment epithelium and choroid," in Excerpta Medica, International Congress Series, K. Shimizu and J. A. Oosterhuis, Eds., pp. 658-662 Elsevier, Amsterdam (1978).

39. L. Feeney-Burns, E. S. Hilderbrand, and S. Eldridge, "Aging human RPE: morphometric analysis of macular, equatorial, and peripheral cells," Invest. Ophthalmol. Vis. Sci. 25, 195-200 (1984).

40. J. J. Weiter, F. C. Delori, G. L. Wing, and K. A. Fitch, "Retinal pigment epithelial lipofuscin and melanin and choroidal melanin in human eyes," Invest. Ophthalmol. Vis. Sci. 27, 145-152 (1986).

41. G. Westheimer, "Dependence of the magnitude of the Stiles-Crawford effect on retinal location," J. Physiol. 192, 309-315 (1967).

42. J. M. Enoch and G. M. Hope, "Directional sensitivity of the foveal and parafoveal retina," Invest. Ophthalmol. 12, 497-503 (1973).

43. H. E. Bedell, "Central and peripheral retinal photoreceptor orientation in amblyopic eyes as assessed by the psychophysical Stiles-Crawford function," Invest. Ophthalmol. Vis. Sci. 19, 49-59 (1980).

44. H. E. Bedell and J. M. Enoch, "A study of the Stiles-Crawford (S-C) function at 35 degrees in the temporal field and the stability of the foveal S-C function peak over time," J. Opt. Soc. Am. 69, 435-442 (1979).

45. P. J. de Lint, T. T. J. M. Berendschot, D. van Norren, "Local photoreceptor alignment measured with a scanning laser ophthalmoscope," Vis. Res. 37, 243-248 (1997).
46. F. C. Delori, D. G. Goger, B. R. Hammond, D. M. Snodderly, and S. A. Burns, "Macular pigment density measured by autofluorescence spectrometry: comparison with reflectometry and heterochromatic flicker photometry," J. Opt. Soc. Am. A 18, 1212-1230 (2001).

47. J. van de Kraats, T. T. J. M. Berendschot, S. Valen, and D. van Norren, "Fast assessment of the central macular pigment density with natural pupil using the macular pigment reflectometer," J. Biomed. Opt. 11, 064031-064037 (2006).

48. T. T. J. M. Berendschot, J. J. M. Willemse-Assink, M. Bastiaanse, P. T. V. M. de Jong, and D. van Norren, "Macular pigment and melanin in age-related maculopathy in a general population," Invest. Ophthalmol. Vis. Sci. 43, 1928-1932 (2002).

49. B. R. Hammond, M. Caruso-Avery, "Macular pigment optical density in a Southwestern sample," Invest. Ophthalmol. Vis. Sci. 41, 1492-1497 (2000).

50. R. A. Bone, J. T. Landrum, and J. C. Gibert, "Macular pigment and the edge hypothesis of flicker photometry," Vis. Res. 44, 3045-3051 (2004).

51. J. Mellerio, S. Ahmadi-Lari, F. van Kuijk, D. Pauleikhoff, A. Bird, and J. Marshall, "A portable instrument for measuring macular pigment with central fixation," Curr. Eye Res. 25, 37-47 (2002).

52. B. R. Wooten, B. R. Hammond, R. I. Land, and D. M. Snodderly, "A practical method for measuring macular pigment optical density," Invest. Ophthalmol. Vis. Sci. 40, 2481-2489 (1999).

53. P. S. Bernstein, F. Khachik, L. S. Carvalho, G. J. Muir, D. Y. Zhao, and N. B. Katz, "Identification and quantitation of carotenoids and their metabolites in the tissues of the human eye," Exp. Eye Res. 72, 215-223 (2001).

54. K. J. Yeum, A. Taylor, G. Tang, and R. M. Russell, "Measurement of carotenoids, retinoids, and tocopherols in human lenses," Invest. Ophthalmol. Vis. Sci. 36, 2756-2761 (1995).

55. T. T. J. M. Berendschot, W. M. R. Broekmans, I. A. Klöpping-Ketelaars, A. F. M. Kardinaal, G. van Poppel, and D. van Norren, "Lens aging in relation to nutritional determinants and possible risk factors for agerelated cataract," Arch. Ophthalmol. 120, 1732-1737 (2002).

56. C. N. Keilhauer and F. C. Delori, "Near-infrared autofluorescence imaging of the fundus: visualization of ocular melanin," Invest. Ophthalmol. Vis. Sci. 47, 3556-3564 (2006).

57. R. W. Knighton, S. G. Jacobson, and C. M. Kemp, "The spectral reflectance of the nerve fiber layer of the macaque retina," Invest. Ophthalmol. Vis. Sci. 30, 2392-2402 (1989).

58. R. W. Knighton and X. R. Huang, "Directional and spectral reflectance of the rat retinal nerve fiber layer," Invest. Ophthalmol. Vis. Sci. 40, 639-647 (1999).

59. Y. Zhang, B. Cense, J. Rha, R. Jonnal, W. Gao, R. Zawadzki, J. Werner, S. Jones, S. Olivier, and D. Miller, "High-speed volumetric imaging of cone photoreceptors with adaptive optics spectral-domain optical coherence tomography," Opt. Express 14, 4380-4394 (2006).

60. W. Gao, R. S. Jonnal, B. Cense, O. P. Kocaoglu, Q. Wang, and D. T. Miller, "Measuring directionality of the retinal reflection with a ShackHartmann wavefront sensor," Opt. Express 17, 23085-23097 (2009).

61. P. de Groot and R. E. Terpstra, "Milimeter-wave model of a foveal receptor," J. Opt. Soc. Am. 70, 1436-1452 (1980). 